\title{
HIF-1a-dynamin2-Kir2.1 pathway contributes to cell proliferation in brain capillary endothelial cells under hypoxic stress
}

\author{
Hideto Yamamura ${ }^{1}$, Yoshiaki Suzuki $^{1}$, Hisao Yamamura ${ }^{1}$, Kiyofumi Asai $^{2}$, Wayne Giles ${ }^{3}$, \\ Yuji Imaizumi ${ }^{1}$
}

\author{
${ }^{I}$ Department of Molecular \& Cellular Pharmacology, Graduate School of Pharmaceutical Sciences, Nagoya City \\ University, Japan, ${ }^{2}$ Department of Molecular Neurobiology, Graduate School of Medical Sciences, Nagoya City \\ University, Japan, ${ }^{3}$ Faculties of Kinesiology and Medicine, University of Calgary, Calgary, Alberta, Canada
}

The blood-brain barrier (BBB) is mainly composed of brain capillary endothelial cells (BCECs), astrocytes and pericytes. The enhancement of BCEC proliferation during brain ischemia may be partly responsible for the ischemia-induced damage of BBB. $\mathrm{Ca} 2+$ signaling, particularly $\mathrm{Ca} 2+$ influx, is a key event of this BCEC enhancement. However, the underlying mechanisms that control $\mathrm{Ca} 2+$ influx are not clear yet. In the present study, t-BBEC117 cells, an immortalized bovine brain endothelial cell line, were cultured under hypoxic conditions at $4 \sim 5 \%$ oxygen for $72 \mathrm{~h}$. This hypoxic stress up-regulated functional expression of Kir2.1 that forms Ba2+-sensitive inward rectifier K+ current in t-BBEC117 cells. Interestingly the expression of Kir2.1 was up-regulated at protein level but not at mRNA level. Hypoxic culture also upregulated dynamin2 expression in t-BBEC117 cells. Fluorescence imaging and co-immunoprecipitation assays revealed the direct interaction between dynamin2 and Kir2.1 channel. When dynamin2 was inhibited by a dynamin inhibitor or dominant-negative form of dynamin2, hypoxia-induced up-regulation of Kir2.1 was canceled. In contrast to many different types of cells, hypoxic culture down-regulated hypoxia-inducible factor-1 $\alpha$ (HIF-1 $\alpha$ ) expression. Knockdown of HIF- $1 \alpha$ increased dynamin 2 expression in t-BBEC117 cells cultured in both normoxic and hypoxic conditions. These results suggest that hypoxia down-regulated HIF-1 $\alpha$ and reciprocally up-regulated dynamin2, resulting in facilitation of Kir2.1 surface expression. In t-BBEC117 cells cultured in hypoxia, store-operated Ca2+ (SOC) entry was enhanced, and this component was partially but significantly reduced by inhibition of Kir2.1. The mRNA expression of SOC channels, such as Orai1, Orai2, and transient receptor potential (TRP) channels, was not affected by the hypoxic stress. MTT assay showed that hypoxia enhanced proliferation of t-BBEC117 cell and this effect was reversed by inhibition of Kir2.1. Our results demonstrated that the hypoxic stress increased Kir2.1 surface expression via HIF-1 $\alpha$-dynamin2 pathway and caused membrane hyperpolarization in BCECs. This hyperpolarization increases $\mathrm{Ca} 2+$ influx and facilitates BCEC proliferation. These signal pathways may be involved in ischemia-induced disruption of BBB (Yamamura H et al., BBRC, 2016). 